\title{
Pathologic Stage II Gastroesophageal Junction Adenocarcinoma AJCC v8
}

National Cancer Institute

\section{Source}

National Cancer Institute. Pathologic Stage // Gastroesophageal Junction

Adenocarcinoma A/CC v8. NCI Thesaurus. Code C133570.

Stage II includes: IIA: (T2, N0, M0, G3); (T2, N0, M0, GX); IIB: (T1, N1, M0, Any G); (T3, N0, M0, Any G). T2: Tumor invades the muscularis propria. T1: Tumor invades the lamina propria, muscularis mucosae, or submucosa. T3: Tumor invades adventitia. N0: No regional lymph node metastasis. N1: Tumor with metastasis in one or two regional lymph nodes. M0: No distant metastasis. GX: Grade cannot be assessed. G3: Poorly differentiated, undifferentiated. (AJCC 8th ed.) 